\title{
How Does a Short, Interrupted Recovery Break Affect Performance and How Is It Assessed? A Study on Acute Effects
}

\author{
Maximilian Pelka, Alexander Ferrauti, Tim Meyer, Mark Pfeiffer, and Michael Kellmann
}

\begin{abstract}
A recovery process with optimal prerequisites that is interrupted is termed disrupted recovery. Whether this process has an influence on performance-related factors needs to be investigated. Therefore, the aim of this study was to examine how a short disturbance of a recovery phase is assessed and whether subsequent repeated-sprint performance is affected by it. A quasiexperimental $2 \times 2$-factor crossover design with 34 sport-science undergraduate students (age $20.3 \pm 2.1$ y) was applied. Factors were the type of intervention (power nap vs systematic breathing; between-subjects) and the experimental condition (disturbed vs nondisturbed break; within-subject). Repeated-sprint performance was measured through $6 \times 4$-s sprint protocols (with 20 -s breaks) before and after a 25-min recovery break on 2 test days. Subjective evaluation of the interventions was measured through the Short Recovery and Stress Scale and a manipulation check assessing whether participants experienced the recovery phase as efficacious and pleasant. Regarding the objective data, no significant difference between sprint performances in terms of average peak velocity $(\mathrm{m} / \mathrm{s})$ on the treadmill was found. The manipulation check revealed that disturbed conditions were rated significantly lower than regular conditions in terms of appreciation, $t_{31}=3.09, P=.01$. Short disturbances of recovery do not seem to affect subsequent performance; nevertheless, participants assessed disturbed conditions more negatively than regular conditions. In essence, the findings indicate a negligible role of short interruptions on an objective level. Subjectively, they affected the performance-related assessment of the participants and should be treated with caution.
\end{abstract}

Keywords: acute recovery, systematic breathing, power naps, monitoring

Breaks by definition are planned or spontaneous recesses from a task that interrupt the task's flow and continuity. ${ }^{1}$ Nevertheless, from an organizational (psychological) point of view, a break may provide a period of free time to recover to guarantee the resumption of work. Research shows that people need occasional changes in the tempo of work or an oscillation between work and recreation, particularly when they are fatigued ${ }^{2}$ or working continuously for an extended period of time. ${ }^{3}$ Apart from the occupational context, periodic breaks in performance also represent a feature of almost every sport. Fixed and scheduled breaks in sports competition and training serve corresponding functions. They allow athletes to recover psychophysically and allow coaches and staff to provide information. ${ }^{4}$ Examples of breaks include the time between 2 training sessions during a training camp (or during regular training situations), between the different disciplines of a decathlon, or between runs in alpine skiing. ${ }^{5}$ Anshel ${ }^{4}$ stated that a halftime is a period during which many issues can be addressed. It includes regrouping, adjusting and reviewing plans and strategies, exchanging information, and recovery. Compared with other types of recesses, these kinds of breaks are positively connoted as they may contribute to recreational or rejuvenating processes in fatigued individuals. ${ }^{1}$ Furthermore, they are a fixed part of the competition and therefore, can be taken into one's considerations, ie, they can be planned in advance. Another

Pelka, Ferrauti, and Kellmann are with the Faculty of Sport Science, Ruhr University Bochum, Bochum, Germany. Kellmann is also with the School of Human Movement and Nutrition Sciences, University of Queensland, Brisbane, Australia. Meyer is with the Inst of Sports and Preventive Medicine, Saarland University, Saarbrücken, Germany. Pfeiffer is with the Inst of Sports Science, Johannes-Gutenberg University, Mainz, Germany. Address author correspondence to Maximilian Pelka at Maximilian.Pelka@ rub.de. type of break with positive effects is labeled a timeout. During a game such as basketball, these spontaneous breaks are called when players need to refocus. The coach's intervention may consist of new instructions, substitutions or to facilitate recovery from fatigue or interrupting the opponents' positive performance and consequent psychological advantage. $^{6}$

Regardless of whether the interruption/break is seen positively or negatively, many practitioners are not aware of the importance of breaks with regard to the vulnerability of athletes in those periods. Especially regarding the recovery aspects of breaks, athletes are still not fully aware of its underlying importance. If rest periods in terms of breaks are not carefully planned they could lead to a deficit in recovery. In case preparations do not occur, impaired subsequent performance is a possible scenario. ${ }^{7}$ Almost 3 decades ago, research revealed that the vulnerability toward interruptions, irritations, and trouble is considerably increased in recovery periods. ${ }^{8}$ Already small changes in homeostasis during those recovery periods may have a great impact on performance and well-being of athletes. Consequences can be characterized as insufficient or disturbed recovery. ${ }^{9}$ Insufficient recovery is defined as the result of overly short recovery periods, which are lacking the prerequisites for adequate recovery. Disturbed recovery is present if prerequisites for optimal recovery are given; the process though is disrupted by environmental issues. ${ }^{10}$ For example, this might involve a waiting area during a long jump event where no shelter is available during a heavy rain shower. The influence of such incidents could be measured via the scale Disturbed Break of the Recovery-Stress Questionnaire for Athletes. ${ }^{11,12}$ However, the issue of disturbed breaks has not been investigated thoroughly, ie, there is no research available on the consequences of disturbances on the assessment of the breaks itself and on the performances that follow these breaks. Because external and internal interruptions are widespread in sports, 
the current study focused on those issues and tried to examine how participants perceived the disturbed recovery break compared with a not disturbed break to see whether that perception has an influence on subsequent performance. The performances were therefore analyzed, as well.

Unpredictable events and changes of conditions are likely to occur, either during recovery from competition or during recovery between competitions. These modifications of situations could stem from internal or external sources, that is, variability in the environmental conditions (eg, noise, heat, rain delay, or problems with facilities), self-related issues such as nervousness, rumination, or even domestic issues (eg, family problems, extra workload) with serious consequences for the affected athlete. ${ }^{7}$ The onset of such an extra activity requires immediate attention thereby interrupting a person on a current task. ${ }^{13}$ Such an incidence may cause frustration, helplessness, or a change in task strategies. ${ }^{14}$ Interruptions tend to have a negative connotation as they are associated with time consuming stops leaving people with insufficient time to accomplish goals. ${ }^{1}$ An additional factor is that those interruptions occur mostly unexpected and interrupt a person's state of total involvement in a task. ${ }^{15}$

Thus, the question of what happens when a break that aimed at inducing recovery and preparing athletes for subsequent performances is interrupted remains. During these moments of deep concentration and attempted relaxation, any disturbance could be experienced as a stressor. Despite those highly relevant concerns, almost no research on those issues in sports context exists. Other disciplines within psychology already focused on that field a few decades ago. A study by Roy ${ }^{16}$ showed that workers with scheduled breaks during their working days managed to focus their attention during the day and even experienced enjoyment. However, when those breaks were interrupted, workdays became almost intolerable. Based on those findings, the aim of the study was to examine whether an interruption of a recovery break negatively influences subsequent sports performance. In addition, we wanted to assess if those interruptions influence the subjective perception of a recovery break and the subjective evaluation of one's own recovery/stress state.

Therefore, the hypotheses were as follows:

- A disturbed break leads to a drop in performance compared with performance followed by a not-disturbed break.

- Disturbed breaks are subjectively assessed as being less relaxing, efficacious, and appreciating and more stressful than regular breaks.

- Participants' mean heart rate will be increased during the disturbed break in comparison with the regular condition.

\section{Methods}

\section{Subjects}

Thirty-four sport-science undergraduate students (age 18-27 y, $20.26 \pm 2.08$ ) volunteered for the study. The sample (23 men, 11 women) engaged in a total of 7 different sport types, from which soccer was most prominent with $35.5 \%$ of participants active. All of the sports included repeated-sprint ability as one of their key factors (handball, basketball, volleyball, tennis, American football, track and field). After a detailed explanation of the benefits and risks of the study, all participants gave their informed consent. The ethics committee of the local faculty of psychology approved the study according to the principles of the Declaration of Helsinki.

\section{Design and Methodology}

A quasi-experimental $2 \times 2$-factor crossover design was applied. The between-subjects factor was the type of intervention (power nap, $\mathrm{n}=18$ vs systematic breathing, $\mathrm{n}=16$ ) whereas the experimental condition (disturbed vs regular recovery; see Figure 1) formed the within-subject factor. To guarantee for an equal gender distribution, male and female participants were separately assigned to one type of intervention; however, within the gender groups, the assignment to the experimental condition was conducted randomly. Participants completed 2 testing days, with one of them randomly chosen to be disturbed in the recovery break (counterbalanced order). These days were scheduled with a 1-week washout period in between to eliminate impressions of the experimental condition. A week before their first experimental session, all participants completed a habituation session during which they were introduced to the facilities and practiced the sprint protocols. The design followed the procedure of a study by Pelka et al, ${ }^{17}$ who found supportive effects for recovery of systematic breathing and power napping between intermittent sprint sessions. Both strategies improved sprinting performance significantly compared with a control group. Based on these results, only breathing regulation and power naps were included as recovery strategies in the current study. A control group was not included, as Pelka et $\mathrm{al}^{17}$ already revealed the significant difference between those 2 intervention strategies and control conditions. Hence, the current study relied on the abovementioned results and used the research design to take a more detailed look at systematic breathing and power napping, thereby focusing on interruptions of the recovery break. During a test day, participants were asked to perform in 2 repeated-sprint sessions, intermitted by a 25 -minute recovery break (Figure 1). The procedure was identical for both testing days, except for the experimental condition (disturbed/regular recovery break).

\section{Recovery}

As previously proven to be supportive in those settings a guided systematic breathing protocol and a power nap were selected as recovery strategies. ${ }^{17}$ The setting of the recovery interventions consisted of a quiet and darkened room. Participants had to lie down and were guided through the prerecorded audio instructions of the systematic breathing or were instructed to engage in a power nap. One of the 2 testing days was randomly chosen to be experimentally manipulated by disturbing the participant's recovery phase. The experimenter entered the room once in the middle of the recovery break and interrupted the participants by checking their heart-rate (HR) monitor while pretending that the instrument showed malfunction. None of the participants indicated to having noticed this deception, which was revealed to them after finishing the study.

\section{Performance and Physiological Data}

Performance was measured on a nonmotorized treadmill (Woodway) through $6 \times 4$-second sprint protocols (with 20-s breaks) before and after the 25-minute recovery breaks (Figure 1). Both sprint sessions were preceded by a warm-up protocol and stretching phase. To guarantee fatigue, another four 4-second sprints were scheduled as a proof to ensure participants were on a high level of physical strain after the first $6 \times 4$-second sprints. ${ }^{17}$ The average peak velocity (in $\mathrm{m} / \mathrm{s}$ ) served as the main performance parameter. In addition, 3 countermovement jumps (CMJs) each had to be executed after 


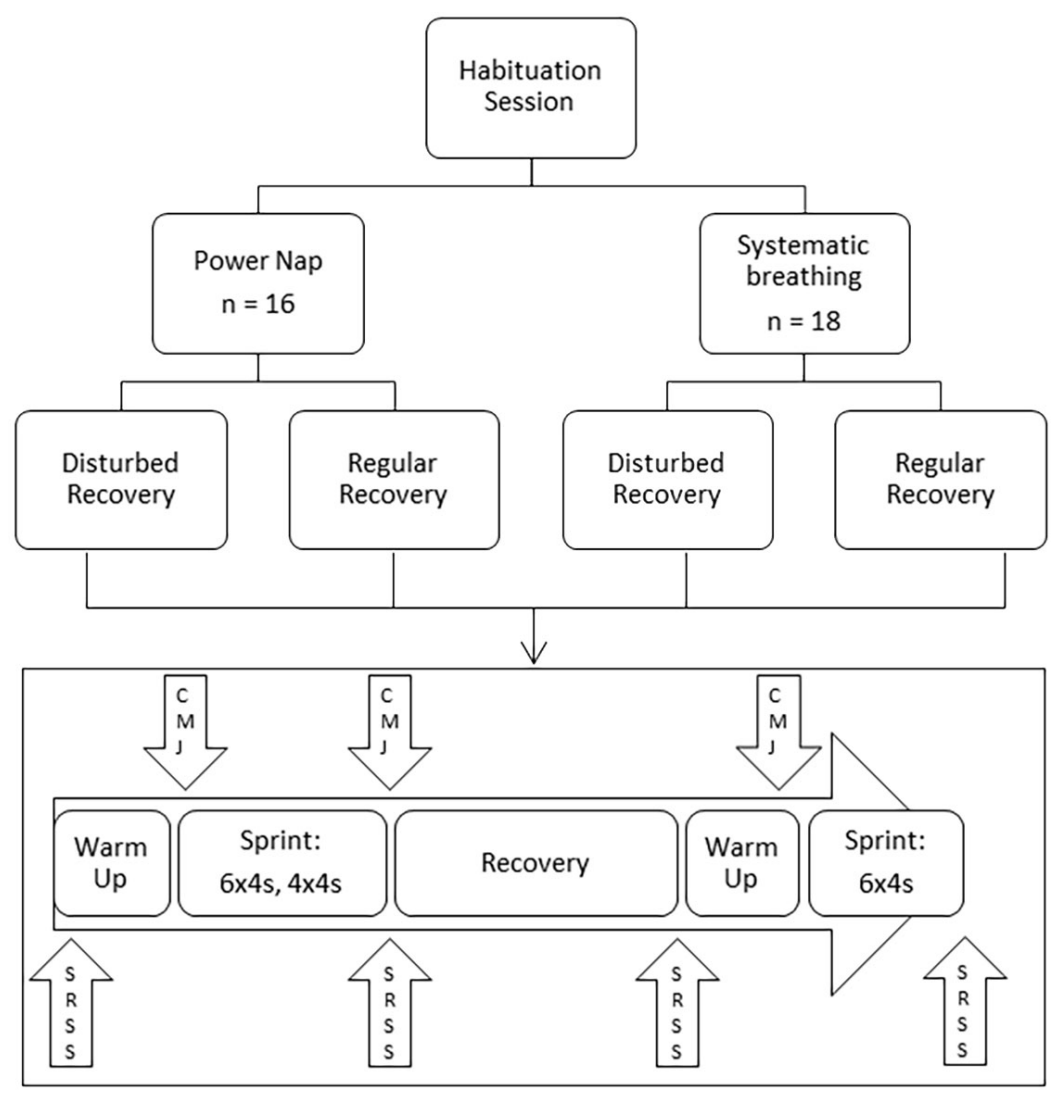

Figure 1 - Study design and course of testing day.

the first warm-up, the first sprint protocol and the second warmup on a contact platform (Haynl Elektronik, Germany). CMJs assessed the physical strain level via jump height through flight time. Participants' HR was recorded during a 5-minute rest before the start of the experiment, during the sprint sessions and during the recovery period.

\section{Subjective Ratings}

Subjective evaluation of the interventions was measured through the Short Recovery and Stress Scale (SRSS) ${ }^{18}$ and a manipulation check that focused on the efficacy and appreciation of the intervention. The SRSS was completed on four occasions during a test day, ie, at the start, after the first sprint protocol, after the recovery break, and after the second sprint protocol (Figure 1). It consists of 8 items, ${ }^{19,20}$ further classified into 4 recovery-related (physical-performance capability, mental-performance capability, emotional balance, and overall recovery) and four stress-related items (muscular stress, lack of activation, negative emotional state, and overall stress). All items are scored independently and were not used to create overall recovery and/or stress scores. Responses had to be stated on a 7-point Likert scale, ranging from does not apply at all (0) to fully applies (6). The manipulation check was completed at the end of both test days. It consists of 2 questions regarding the efficacy ("How effective do you think the recovery break was?") and appreciation ("How much did you like the recovery break?") of the recovery phase. Answers had to be indicated on a 7-point Likert scale ranging from 0 (not at all) to 6 (very much), as well.

\section{Statistical Analysis}

Statistical analyses were conducted using SPSS 22 (IBM, 2012) and involved repeated-measures analyses of variance (RMANOVA) to identify effects of the type of recovery intervention and of their implementation on stress and recovery. If assumptions of sphericity were violated in one of the RM-ANOVA, Greenhouse-Geisser corrections were used. If significant results $(P \leq .05)$ were obtained, significance and mode of the effect were further analyzed via Bonferroni-corrected post hoc tests if appropriate. Following Cohen's ${ }^{21}$ argumentation on manipulated variables effect sizes were reported using partial eta squared $\left(\eta_{\mathrm{p}}{ }^{2}\right)$. Values can be evaluated as small (0-.029), medium (.03-.159), and large $(.16-.35){ }^{22}$

\section{Results}

\section{Performance and Physiological Data}

Sprint Output. Regarding the performance data, no significant difference between sprint performances in terms of average peak velocity $(\mathrm{m} / \mathrm{s})$ on the treadmill was found through $2 \times 2 \times 2 \mathrm{RM}$ ANOVA (manipulation $\times$ intervention $\times$ sprint) between the 2 manipulation conditions (disturbed vs regular recovery), $F_{1,30}=.46$, $P=.50, \eta_{\mathrm{p}}^{2}=.02$ (see Table 1 for means, standard deviations, and $95 \%$ confidence intervals). In addition, no significant differences between the first and second sprint session of a test day occurred, $F_{1,30}=.12, P=.73, \eta_{\mathrm{p}}^{2}=.01$. The same applies for the interaction between manipulation conditions and measurement times, $F_{1,30}=$ 
$.43, P=.52, \eta_{\mathrm{p}}{ }^{2}=.02$. Finally, the type of intervention (power nap vs breathing) did not affect the results significantly.

Countermovement Jumps. The results of a second $2 \times 2 \times 3$ RM-ANOVA (manipulation $\times$ intervention $\times$ jump) on the CMJ data revealed a significant effect on jump height throughout the three measurement points, $F_{2,64}=37.41, P<.001, \eta_{\mathrm{p}}{ }^{2}=.54$. Table 2 shows that CMJs were lower after the sprints compared with both CMJs before sprint session 1 and before sprint session 2. There was no further significant difference between manipulation conditions, $F_{1,32}=2.07, P=.16, \eta_{\mathrm{p}}{ }^{2}=.06$, or an interaction effect, $F_{2,64}=.33$, $P=.72, \eta_{\mathrm{p}}{ }^{2}=.01$. As for the sprint output, the type of intervention did not influence the performance.

Heart Rate. Regarding the analysis of the mean HR $(2 \times 2 \times 4$ RMANOVA; manipulation $\times$ intervention $\times$ recording time) it can be stated that there was a significant difference between measurement points over time, $F_{3,90}=980.75, P<.001, \eta_{\mathrm{p}}{ }^{2}=.97$. In addition, no differences were found between manipulations, $F_{1,30}=.0^{\prime}{ }_{2}{ }^{\prime}=.77$, $\eta_{\mathrm{p}}^{2}=.01$, or an interaction effect, $F_{3,90}=.73, P=.53, \eta_{\mathrm{p}}^{2} \eta_{P}^{2}=.02$. Exact means, standard deviations, and $95 \%$ confidence intervals are presented in Table 3. Considering the peak HR during the recovery break, a significant difference between manipulation conditions during the recovery break was obtained, $t_{30}=-4.20, P<.001,95 \%$ CI $[-14.72 ;-5.09]\left(M_{\text {not disturbed }}=119.42 \pm 12.66 ; M_{\text {disturbed }}=129.32\right.$ $\pm 14.94)$. A significant interaction effect for manipulation $\times$ time in a RM ANOVA was found before post hoc tests were performed, $F_{1.74,48.62}=4.33, P<.02, \eta_{\mathrm{p}}^{2}=.13$.

\section{Subjective Ratings}

Analyses of the SRSS scales $(2 \times 2 \times 4$ RM-ANOVA; manipulation $\times$ intervention $\times$ time of completion) revealed that the values of overall stress and overall recovery differed significantly over time, $F_{3,81}=$ $39.67, P<.001, \eta_{\mathrm{p}}{ }^{2}=.60$ (overall recovery) $F_{3,81}=34.42, P<.001$, $\eta_{\mathrm{p}}{ }^{2}=.58$ (overall stress). For both scales, manipulation conditions and interactions effects did not become significant; respective $95 \%$ confidence intervals can be found in Table 4 . The same pattern was evident for the other 6 scales, as well; that is, changes over time were significant and differences between manipulation conditions and interactions were not. Regarding the manipulation check, experienced appreciation was significantly different between disturbed and regular recovery conditions. Disturbed conditions were rated significantly lower (ie, less appreciated) than nondisturbed conditions, $t_{31}=3.09, P=.01, d=.40$. The efficacy of the recovery

Table 1 Average Peak Velocity $(\mathrm{m} / \mathrm{s})$ During Both Sprint Sessions

\begin{tabular}{lcccc}
\hline Recovery & Sprint 1 & 95\% Cl & Sprint 2 & 95\% Cl \\
\hline Disturbed & $5.06 \pm .65$ & {$[4.84,5.29]$} & $5.03 \pm .65$ & {$[4.81,5.26]$} \\
Regular & $5.10 \pm .63$ & {$[4.89,5.33]$} & $5.13 \pm .61$ & {$[4.92,5.35]$} \\
\hline
\end{tabular}

Note: As there were no significant differences between intervention groups, Tables 1-5 focus on the experimental condition (disturbed vs regular recovery). breaks was rated marginally different, $t_{31}=1.76, P=.08, d=.25$. Participants rated the disturbed condition lower (ie, less efficacious) than the regular recovery condition (see Table 5). Furthermore, the data for appreciation showed that male participants experienced the disturbed conditions significantly worse, that is, more negative, than female participants did, $F_{1,30}=9.24, P=.01, \eta_{\mathrm{p}}^{2}=.24$. Both SRSS and manipulation check, were not significantly influenced by intervention conditions.

\section{Discussion}

The benefit of breaks in sport (eg, halftime, timeout) is the psychophysical regeneration of athletes. Interruptions that disturb these breaks unexpectedly are perceived negatively as they are associated with increased physiological arousal and negative affect. Based on these assumptions, the current study aimed at assessing the acute effects of an interruption of a fixed break on subsequent performance together with the subjective evaluation of the break. Our results indicate that short interruptions of recovery breaks do not affect subsequent repeated-sprint performance. However, the interruption partly affected the assessment of the recovery break, as participants evaluated the interrupted recovery break as being less pleasing than the regular break. Moreover, our analyses revealed greater male aversion toward the interruption compared with females. Regarding the validity of the study, the results of the CMJ, HR, and SRSS data underline the need for recovery, as all those measures reacted sensitively in accordance to the demands of the sprinting task and the stress and recovery experienced. For example, the scores on the stress-related items of the SRSS went up after the sprints and down after the recovery period. Reversely, the scores of the recovery-related items decreased after the sprints and increased after recovery. CMJ performance was better after the recovery period compared with the performance after the sprints. The 2 manipulation conditions (disturbed vs regular recovery), as well as the 2 intervention conditions (systematic breathing vs power nap) showed similar patterns over time on CMJ, HR, and SRSS measures.

A set of repeated sprints is highly demanding for an athlete and induces fatigue on multiple parameters. ${ }^{23}$ First, focusing on performance-related variables, fatigue is operationalized through a reduction in speed or maximal power output. ${ }^{23}$ Second, concerning the autonomic nervous system, parasympathetic reactivation is highly impaired after repeated sprinting ${ }^{24}$ and therefore the organism is not capable of conserving energy. Third, as a response to physiological stress, hormone (eg, cortisol) and cytokine (eg, interleukin-6) secretion is increased and results in a heightened inflammatory response. ${ }^{25}$ To support the recovery process and to enhance subsequent performance between two repeated-sprint bouts, two recovery strategies were implemented in the recovery break between those two bouts in the current study. Previous research has shown that the strategies implemented in the current study, systematic breathing and napping, have been proven to be supportive and performance enhancing. ${ }^{17,26}$ Specifically, systematic

Table 2 Countermovement Jumps (cm) Before and After Sprint and Recovery Sessions

\begin{tabular}{lcccccc}
\hline Recovery & Before sprint 1 & $95 \% \mathbf{C l}$ & Before recovery & 95\% Cl & After recovery & 95\% Cl \\
\hline Disturbed & $34.74 \pm 5.62$ & {$[32.77,36.70]$} & $32.65 \pm 4.74$ & {$[30.99,34.30]$} & $34.96 \pm 5.14$ & {$[33.17,36.75]$} \\
Regular & $35.79 \pm 5.14$ & {$[33.72,37.87]$} & $33.27 \pm 5.47$ & {$[31.36,35.18]$} & $35.50 \pm 6.41$ & {$[33.27,37.74]$} \\
\hline
\end{tabular}




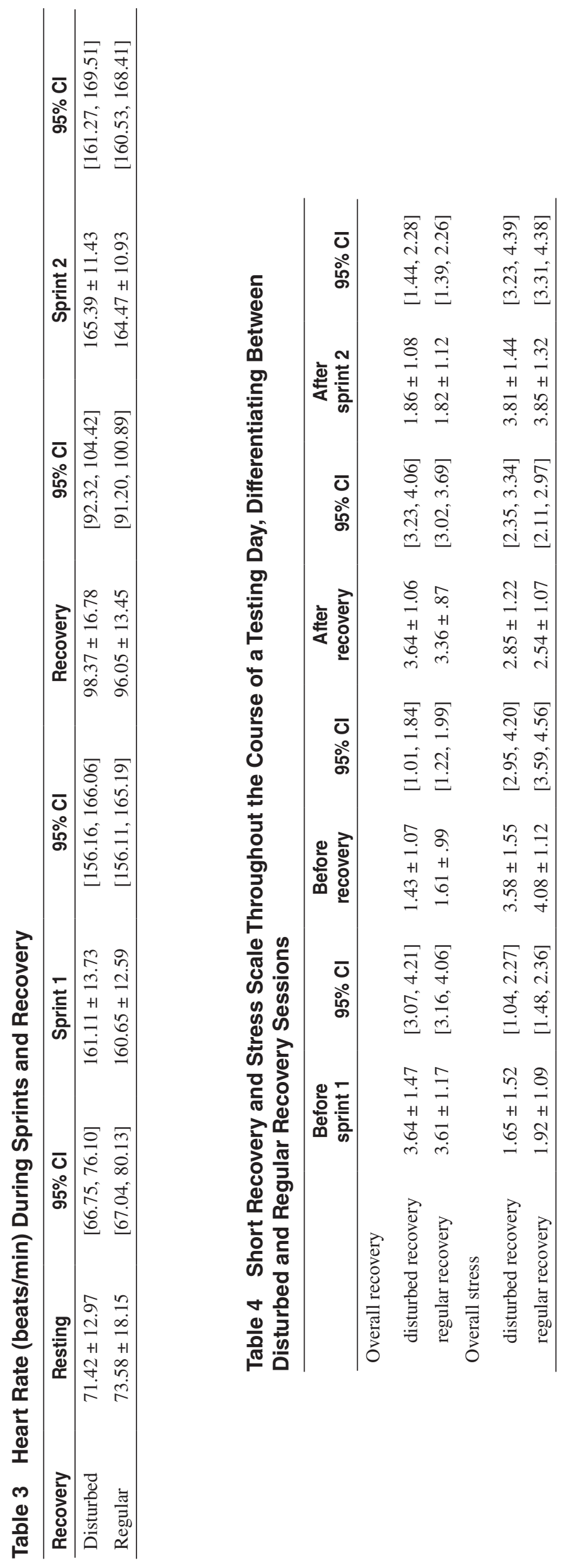


Table 5 Manipulation Check After Disturbed and Regular Recovery Testing Days

\begin{tabular}{lcccc}
\hline Recovery & Efficacy & $\mathbf{9 5 \%} \mathbf{C l}$ & Appreciation & $95 \% \mathbf{C l}$ \\
\hline Disturbed & $4.16 \pm 1.14$ & {$[3.75,4.57]$} & $4.09 \pm 1.12$ & {$[3.69,4.50]$} \\
Regular & $4.41 \pm .88$ & {$[4.09,4.72]$} & $4.53 \pm 1.08$ & {$[4.14,4.92]$} \\
\hline
\end{tabular}

breathing is known to increase parasympathetic nervous system activity and lower sympathetic nervous system activity ${ }^{27,28}$ in terms of an increase in respiratory functioning, exercise tolerance or a decrease of dyspnea. ${ }^{27}$ Napping (less than $30 \mathrm{~min}$ ) has been shown to improve motor and sprinting performance ${ }^{17,26}$ and also to influence hormone (eg, cortisol distribution) and cytokine secretion (eg, interleukin-6 suppression). ${ }^{29,30}$ Vgontzas et al ${ }^{30}$ reported that cortisol levels dropped significantly compared with a control group during naps and increased significantly different during the postnap phase. This specific behavior of cortisol concentrations is associated with an increased energy supply after the recovery break, which could lead to improved performance. A second contributor to improved performance was the suppression of interleukin-6 secretion during and after naps. ${ }^{30}$ Through those physiological and hormonal changes, an increase in alertness/readiness can be explained and postnap performance potentially predicted.

Hypothetically, those regeneration processes should have been interrupted by the disturbance during the recovery break. It was expected that the interruption should have explicitly led to a heightened autonomic nervous system response and increased feelings of stress. ${ }^{31}$ These responses can be operationalized via various parameters, for example, HR, skin conductance levels breathing rate, and peripheral vasoconstriction. Regarding these psychophysiological reactions, the current study used HR as a measure, but differences in mean HR between the manipulation conditions during the recovery break could not be detected. However, a significant difference in peak HR (maximal HR that was measured during the specific time frame) was measured; that is, the peak HR in the disturbed recovery condition was 9 beats/min higher than in the regular recovery condition. Thus, even though acute physiological changes during the recovery periods were found, the overall (average) recovery period was not affected.

In contrast to the sports context, the effects of interrupted breaks have already been of interest in industrial and organizational psychology. ${ }^{7,14,16}$ Fisher $^{14}$ assumes that interruptions would be more irritating if they were very frequent, the task has been enjoyed, or they were uncontrollable. Two of those could be applied to the experimental condition of the current study as participants, first, had no control over the interruption, and, second, appreciated the recovery break (a mean of 4.5 points on a scale ranging up to 6 in the manipulation check). As the recovery break was only interrupted once during the current study Fisher's first assumption may explain why participants were not irritated sufficiently to influence performance. Beyond acute effects on performance, considering potential long-term effects of disturbed breaks on well-being, Laux et $\mathrm{al}^{32}$ found that acute stress in combination with occurrence of disturbed breaks may contribute to injury risk. Their study revealed a direct and significant relationship between heightened injury risk and disturbed breaks, as they implemented the Recovery-Stress Questionnaire for Athletes throughout an entire season in a professional football club. ${ }^{32}$ Following these authors, breaks have to be monitored as well as training and competition. They concluded that it may even be that the individual perception of recovery rather than the objective length is the crucial variable. ${ }^{32}$

\section{Practical Applications and Limitations of the Study}

Although ecological validity is higher for high performance sports when examining such effects with Olympic or elite athletes, our aim was to identify basic mechanisms. Therefore, we chose a sport-student sample in a laboratory-based experiment to control variables of interest, ie, break length and performance execution. However, as conclusions for sports practice should be drawn from the present and subsequent studies, environmental factors need to be taken into account more thoroughly for research on a professional and international sport level. A variety of interruptions such as background noise, rain delays, or conversations of others might interrupt concentration and recovery, ${ }^{33}$ yet they might be perceived differently and trigger different reactions in a laboratory context compared with the European Championship or Olympic finals. During the latter situations, more is at stake for the individual and demands are higher. Therefore, generalizing from one situation to others might not be suitable and needs to be investigated, both quantitatively and qualitatively.

One critical aspect that needs to be addressed is the fact that we were not able to fully ensure that participants actually followed the instructions of the respective condition. One of the study's main goals was to keep the conditions as natural as possible; therefore, we did not attach more than a HR monitor to the participants. Consequently, based on HR data alone, it is not possible to draw conclusions as accurate as if they were based on EEG and/or biofeedback systems. A second factor contributing to the natural character of the recovery break was that participants were left alone in the testing room. This was a major difference compared with the study of Pelka et al, ${ }^{17}$ who reported using personally supervised instructions. Third, in the current study prerecorded audio instructions were used in the systematic breathing condition. This was administered particularly to keep the situation as normal as possible by not disturbing participants with an unfamiliar person. Using audio instructions is standard practice and the majority of research reports its usage. ${ }^{34,35}$ In addition, to preempt this issue, the authors conducted a prestudy in which a supervised breathing condition was compared with an audio condition in exactly the same research design. This study revealed no significant performance differences after audio or supervised personal instructions. However, when comparing the results of Pelka et al ${ }^{17}$ with the data of the current study, the mean HR values during the recovery break in the current study were ten bpm higher on average. This finding might indicate that recovery was not sufficient enough to affect subsequent sprinting performance.

Similar to the systematic breathing condition, it is not confirmed whether participants slept during their recovery break. However, following previous research, it was suggested that healthy subjects are able to sleep in a relatively short period of time when appropriate conditions have been created. ${ }^{36}$ It was further expected that a 25-minute break would allow approximately 10 to 20 minutes of sleep to be achieved. ${ }^{37}$ In line with these findings, available data on nap efficacy report benefits from naps of 10 to 15 minutes in length. ${ }^{38}$ If those durations were not achieved, the lack of sleep during the recovery break could have influenced the effectiveness of the power nap condition. This might explain the stagnated performance after the power nap condition. Hayashi et $\mathrm{al}^{39}$ proposed 
that 3 minutes of stage 2 sleep have recuperative effects; whereas these effects are limited after only stage 1 sleep and nonexistent if participants did not sleep at all. Subjective alertness and performance in a visual detection task increased only after stage 2 phase was experienced. Sleep including only stage 1 sleep and not napping led to significantly worse performance. To provide an in-depth view in this regard, follow-up studies need to consider physiological and hormonal changes thoroughly. Additional physiological data need to be gathered to allow for more accurate conclusions. Except for the HR data, we did not measure more physiological parameters of the participants. This has to be established while keeping the experimental conditions as natural as possible to obtain ecologically valid data.

\section{Conclusions}

In conclusion, a short interruption of a recovery break between 2 repeated-sprint sessions did not affect objective variables associated with the sprint performance. Furthermore, no effects for the subjective outcomes (in terms of the SRSS) could be demonstrated in this study. Although participants recognized the difference between the manipulation conditions and rated the not disturbed condition as being more pleasant, they did not perceive a difference in efficacy. Changes in the design of the recovery interruption, as well as different levels of athletes, should be considered in future studies to investigate the issue of disturbed breaks in more detail.

\section{Acknowledgments}

This work was funded by the German Federal Institute for Sport Science (Grant No. IIA1-081901/12-16).

MP conceived, designed, and conducted the study. MK supervised the study. MP drafted the manuscript. MP, AF, TM, MP, and MK critically revised the manuscript for important intellectual content. All authors approved the final version of the manuscript. Furthermore, we would like to thank Birte Thissen for her input and support during the experimental and data preparation phase.

\section{References}

1. Jett Q, George J. Work interrupted: a closer look at the role of interruptions in organizational life. Acad Manage Rev. 2003;28:494-507.

2. Henning RA, Sauter S, Salvendy G, et al. Microbreak length, performance, and stress in a data entry task. Ergonomics. 1989;32:855-864. PubMed doi:10.1080/00140138908966848

3. Csikszentmihalyi M. Play and intrinsic rewards. J Humanist Psychol. 1975;15(3):41-63.

4. Anshel M. Sport Psychology: From Theory to Practice. Scottsdale, AZ: Gorsuch Scaribrick; 1990.

5. Wrisberg C, Anshel M. The use of positively-worded performance reminders to reduce warm-up decrement in the field hockey penalty shot. J Appl Sport Psychol. 1997;9(2):229-240. doi:10.1080/10413209708406484

6. Gómez M, Jiménez S, Navarro R, et al. Effects of coaches' timeouts on basketball teams' offensive and defensive performances according to momentary differences in score and game period. Eur J Sport Sci. 2011;11(5):303-308. doi:10.1080/17461391.2010.512366

7. Kallus K, Eberspächer H, Hermann H. Systematische, naive und gestörte Regeneration im Sport. In: Montada L, ed. Bericht über den 38 Kongress der Deutschen Gesellschaft für Psychologie in Trier. Göttingen, Germany: Hogrefe; 1992:436-437.
8. Wilhelm A, Janssen J. Beanspruchung und Belastung im Triathlon [Stress in triathlon]. Sportpsychologie. 1989;3:18-22.

9. Kellmann M. Underrecovery and overtraining: different conceptssimilar impact? In: Kellmann M, ed. Enhancing Recovery: Preventing Underperformance in Athletes. Champaign, IL: Human Kinetics; 2002:3-24.

10. Kellmann M, Altenburg D, Lormes W, et al. Assessing stress and recovery during preparation for the world championships in rowing. $T$ Sport Psychol. 2001;15:151-167. doi:10.1123/tsp.15.2.151

11. Kellmann M, Kallus KW. The Recovery-Stress Questionnaire for Athletes. In: Kallus KW, Kellmann M, eds. The Recovery-Stress Questionnaires: User Manual. Frankfurt am Main, Germany: Pearson Assessment \& Information GmbH; 2016:86-131.

12. Kellmann M, Kallus KW. The Recovery-Stress Questionnaire for Athletes. Champaign, IL: Human Kinetics; 2001.

13. Cellier J, Eyrolle H. Interference between switched tasks. Ergonomics. 1992;35:25-36. doi:10.1080/00140139208967795

14. Fisher C. Effects of external and internal interruptions on boredom at work: two studies. J Organ Behav. 1998;19:503-522. doi:10.1002/ (SICI)1099-1379(199809)19:5<503::AID-JOB854>3.0.CO;2-9

15. Carver C, Scheier M. Origina and functions of positive and negative affect: a control-process view. Psychol Rev. 1990;97(1):19-35. doi:10.1037/0033-295X.97.1.19

16. Roy D. Banana time: job satisfaction and informal interaction. Hum Organ. 1960;18:156-158.

17. Pelka M, Kölling S, Ferrauti A, et al. Acute effects of psychological relaxation techniques between two physical tasks. J Sports Sci. 2016;35(3):216-223. doi:10.1080/02640414.2016.1161208

18. Kellmann M, Kölling S, Hitzschke B. Das Akutmaß und die Kurzskala zur Erfassung von Erholung und Beanspruchung im Sport-Manual [The Acute Measure and the Short Scale of Recovery and Stress for Sports—Manual]. Hellenthal, Germany: Sportverlag Strauß; 2016.

19. Hitzschke B, Kölling S, Ferrauti A, et al. Entwicklung der Kurzskala zur Erfassung von Erholung und Beanspruchung im Sport (KEB) [Development of the Short Recovery and Stress Scale for Sports (SRSS)]. Z Sportpsychol. 2015;22(4):146-162. doi:10.1026/1612-5010/a000150

20. Kölling S, Hitzschke B, Holst T, et al. Validity of the Acute Recovery and Stress Scale - training monitoring of the German junior national field hockey team. Int J Sports Sci Coaching. 2015;10:529-542. doi:10.1260/1747-9541.10.2-3.529

21. Cohen J. Eta-squared and partial eta-squared in fixed factor ANOVA designs. Educ Psychol Meas. 1973;33:107-112. doi:10.1177/ 001316447303300111

22. Cohen J. A power primer. Psychol Bull. 1992;112:155-159. PubMed doi:10.1037/0033-2909.112.1.155

23. Girard O, Mendez-Villanueva A, Bishop B. Repeated-sprint ability_part I. Sports Med. 2011;41(8):673-694 doi:10.2165/11590550000000000-00000. PubMed

24. Buchheit M, Laursen PB, Ahmaidi S. Parasympathetic reactivation after repeated sprint exercise. Am J Heart Circ Physiol. 2007;293:H133H141. PubMed doi:10.1152/ajpheart.00062.2007

25. Abedelmalek S, Souissi N, Chtourou H, et al. Effects of sleep deprivation on proinflammatory cytokines, growth hormone, and steroid hormone concentrations during repeated brief sprint interval exercise. Chronobiol Int. 2013;30(4):502-509. PubMed doi:10.3109/07420528.2012.742102

26. Waterhouse J, Atkinson G, Edwards B, et al. The role of short post-lunch nap in improving cognitive, motor, and sprint performance in participants with partial sleep deprivation. J Sports Sci. 2007;25(14):15571566 doi:10.1080/02640410701244983. PubMed

27. Gosselink R. Controlled breathing and dyspnea in patients with chronic obstructive pulmonary disease (COPD). J Rehabil Res Dev. 2003;40(5):25-33. PubMed doi:10.1682/JRRD.2003.10.0025 
28. Holland AE, Hill CJ, Jones AY, McDonald CF. Breathing exercises for chronic obstructive pulmonary disease. Cochrane Database Syst Rev. 2012;10: doi:10.1002/14651858.CD008250.pub2. PubMed

29. Maschke C, Hecht K. Stress hormones and sleep disturbances-electrophysiological and hormonal aspects. Noise Health. 2004;6:49-54. PubMed

30. Vgontzas AN, Pejovic S, Zoumakis E, et al. Daytime napping after a night of sleep loss decreases sleepiness, improves performance, and causes beneficial changes in cortisol and interleukin-6 secretion. Am J Physiol Endocrinol Metab. 2007;292(1):E253-E261. doi:10.1152/ ajpendo.00651.2005

31. MacDowell K, Mandler G. Constructions of emotion: discrepancy, arousal, and mood. Motiv Emot. 1989;13(2):105-124. doi:10.1007/ BF00992957

32. Laux P, Krumm B, Diers M, et al. Recovery-stress balance and injury risk in professional football players: a prospective study. J Sports Sci. 2015;33(20):2140-2148. PubMed doi:10.1080/02640414.2015.1064 538

33. Nicholls AR, Polman RC. Coping in sport: a systematic review. J Sports Sci. 2007;25(1):11-31. PubMed doi:10.1080/02640410600630654

34. Tsai HJ, Kuo TB, Lee G-S, Yang CC. Efficacy of paced breathing for insomnia: enhances vagal activity and improves sleep quality.
Psychophysiology. 2015;52(3):388-396 doi:10.1111/psyp.12333. PubMed

35. Bing-Canar H, Pizzuto J, Compton RJ. Mindfulness-of-breathing exercise modulates EEG alpha activity during cognitive performance [published online ahead of print June 1, 2016]. Psychophysiology. doi:10.1111/psyp. 12678

36. Kayumov L, Rotenberg V, Buttoo K, et al. Interrelationships between nocturnal sleep, daytime alertness, and sleepiness: two types of alertness proposed. J Neuropsychiatry Clin Neurosci. 2000;12:86-90. PubMed doi:10.1176/jnp.12.1.86

37. Chang Y-S, Wu Y-H, Lu MR, et al. Did a brief nap break have positive benefit son information processing among nurses working on the first 8-h night shift? Appl Ergon. 2015;48:104-108. PubMed doi:10.1016/j. apergo.2014.11.005

38. Takahashi M, Fukuda H, Arito H. Brief naps during post-lunch rest: effects on alertness, performance, and autonomic balance. Eur J Appl Physiol. 1998;78:93-98. PubMed doi:10.1007/s004210050392

39. Hayashi M, Motoyoshi N, Hori T. Recuperative power of a short daytime nap with or without stage 2 sleep. Sleep. 2005;28(7):829-836. PubMed 